ORIGINAL ARTICLE

\title{
Multiorgan dysfunction in infants with post-asphyxial hypoxic-ischaemic encephalopathy
}

\author{
P Shah, S Riphagen, J Beyene, M Perlman
}

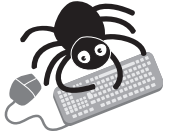

This is an abridged version of a longer version which can be found at www.archdischild.com/ supplemental

See end of article for authors' affiliations .....................

Correspondence to: Dr Shah, Room 775A, Department of Paediatrics, Mount Sinai Hospital, 600 University Avenue, Toronto, Ontario, Canada M5G 1X5;

pshah@mtsinai.on.ca

Accepted 12 March 2003

Background: Multiorgan dysfunction (MOD) is one of four consensus based criteria for the diagnosis of intrapartum asphyxia. The theoretical concept behind MOD is the diving reflex (conservation of blood flow to vital organs at the cost of non-vital organs).

Objectives: To assess the patterns of involvement of each major organ/system and combinations of involvement in infants with post-asphyxial hypoxic-ischaemic encephalopathy (HIE), and to describe this in relation to long term outcome.

Design: Retrospective cohort study.

Setting: Regional tertiary neonatal intensive care unit at the Hospital for Sick Children, Toronto, Canada. Patients: Term neonates with post-intrapartal asphyxial HIE assessed for kidney, cardiovascular system, lung, and liver function.

Outcome: Death and presence or absence of severe neurodevelopmental disability.

Results: Out of 130 of 144 eligible infants with outcome data, $80(62 \%)$ had severe adverse outcome and $50(38 \%$ ) had good outcome. All infants had evidence of MOD (at least one organ dysfunction in addition to HIE). Renal, cardiovascular, pulmonary, and hepatic dysfunction was present in $58-88 \%$ of infants with good outcome and $64-86 \%$ of infants with adverse outcome.

Conclusions: MOD was present in all the infants with severe post-asphyxial HIE. However, there was no association between MOD and outcome in these infants. No relation between individual or combinations of organ involvements and long term outcomes was observed.

$\mathrm{T}$ here is consensus of opinion of representative obstetric and paediatric associations that multiorgan or multisystem dysfunction (MOD) is a constant feature of the neonatal post-asphyxial syndrome. ${ }^{12}$ The MOD phenomenon is mechanistically related to the diving reflex. The reflex, activated by asphyxia, consists of shunting blood from the skin and splanchnic area to the heart, adrenals, and brain, ostensibly to protect these vital organs from hypoxicischaemic injury. ${ }^{3-6}$ Thus, it is likely that each neonate with clinically detectable heart or brain dysfunction resulting from intrapartum asphyxia would have activated the diving reflex for long enough to cause dysfunction of one or more nonessential organs, particularly kidney and liver. This is expected especially in neonates who prove to have permanent brain injury.

The presence of MOD in every neonate with post-asphyxial hypoxic-ischaemic encephalopathy (HIE) has been questioned by some authors. ${ }^{7}$ Inconsistencies in the involvement of the various organs would support the notion that the diving reflex is inconsistently activated in the human, as is the case in animal models of asphyxia. ${ }^{6}$ If MOD is not consistently present in infants with severe HIE, then the dependability of the protection afforded by the diving reflex would be questionable. The patterns of MOD have not been studied in relation to the long term neurological outcome of human infants who sustained post-intrapartum asphyxial HIE of variable severity, and who had had at least four organs/systems tested in addition to the central nervous system.

The objective of the study was to assess the patterns of involvement of each major organ/system and combinations of organs/systems in infants with post-asphyxial HIE, and to describe the associations between the dysfunction of each organ/system and long term outcome.

\section{PATIENTS AND METHODS Infants}

The infants were admitted between 1985 and 1995 to the regional neonatal intensive care unit at the Hospital for Sick Children, Toronto, Canada. All infants were born in peripheral hospitals in and around Toronto (total number of live births about 600000 during the 11 year period) at full term.

\section{Eligibility criteria}

The following eligibility criteria were adapted from the statements of the American College of Obstetricians and Gynecologists and the Society of Obstetricians and Gynecologists of Canada ${ }^{129}$ for ascertaining the presence of post-intrapartum asphyxial HIE:

(1) One or more of the following:

(a) five minute Apgar score of $<5$

(b) metabolic acidosis (cord arterial blood or blood gas analysis within first hour after birth) indicated by a base deficit $\geqslant 16 \mathrm{mmol} / \mathrm{l}$

(c) delayed onset of respiration for five or more minutes

(2) Need for mechanical ventilation at birth

(3) Evidence of encephalopathy including altered state of consciousness and/or seizures (seizures were defined retrospectively from the description provided in the health records

Abbreviations: HIE, hypoxic-ischaemic encephalopathy; MOD, multiorgan dysfunction 
using Volpe's criteria and were mostly subtle, tonic, and tonic clonic types $)^{10}$

(4) Infants who had complete clinical and/or investigational assessments of the function of all four organs as outlined below

Infants with missing data for criterion 1 were included if they were born by emergency caesarean section and had features typical of criteria 2 and 3 and other causes of neonatal encephalopathy could be excluded with confidence.

\section{Exclusion criteria}

Infants were excluded if they were born preterm $(<37$ weeks postmenstrual age), had congenital abnormalities including subtle dysmorphism of unknown significance or a major anomaly of a single organ, inborn errors of metabolism, congenital viral infections, haemorrhagic shock without evidence of intrapartum asphyxia, septic shock, cranial birth trauma, meconium aspiration syndrome, or evidence of antepartum asphyxia. The criteria for antepartum asphyxia were one or more of the following: a history of an antepartum episode of loss of fetal movements lasting for 24 hours or more, severe intrauterine growth retardation (birth weight below the mean for sex and gestational age minus $2 \mathrm{SD}$ ), oligohydramnios, or lack of fetal heart rate variability on admission of the mother to hospital.

\section{Criteria for organ/system dysfunctions}

The criteria for involvement of each organ/system were as follows:

- Renal: anuria or oliguria $(<1 \mathrm{ml} / \mathrm{kg} / \mathrm{h})$ for 24 hours or more, and a serum creatinine concentration $>100 \mathrm{mmol} /$ l; or anuria/oliguria for $>36$ hours; or any serum creatinine $>125 \mathrm{mmol} / \mathrm{l}$; or serial serum creatinine values that increased postnatally

- Cardiovascular: hypotension treated with an inotrope for more than 24 hours to maintain blood pressure within the normal range, or electrocardiographic evidence of transient myocardial ischaemia

- Pulmonary: need for ventilator support with oxygen requirement $>40 \%$ for at least the first four hours after birth

- Hepatic: aspartate aminotransferase > $100 \mathrm{IU} / \mathrm{l}$ or alanine aminotransferase $>100 \mathrm{IU} / \mathrm{l}$ at any time during the first week after birth

\section{Adverse outcome}

Patients were considered to have severe adverse outcome if any of the following occurred:

(1) Death attributable to post-asphyxial HIE

(2) Severe cerebral palsy diagnosed by 12 months of age

(3) Mild or moderate cerebral palsy with blindness or deafness diagnosed by 12 months of age

(4) Moderate cerebral palsy with suspected developmental delay at 12 months of age, confirmed by a Bayley score lower than 2 SD below the mean at 21-24 months of age.

\section{Ascertainment of outcomes}

Outcome data were determined from the records of neonatal follow up and neurology clinics and from re-admissions. The clinicians conducting follow up examinations and authors reviewing health records for multiorgan data respectively were not blinded to the extent of MOD and the outcome of each subject. These potential sources of bias were minimised by our relatively objective outcome criteria. Where follow up was incomplete, a letter requesting a telephone interview was mailed to the family after the family doctor or paediatrician had been asked if this would be appropriate. Outcome data were obtained from the family doctor or paediatrician in seven such cases. The research ethics board of the Hospital for Sick Children approved the study.

\section{Statistical analysis}

For analyses, infants were grouped by long term outcome (adverse versus good outcome). Continuous variables were compared between the two outcome groups using the two tailed Student's $t$ test. The incidence of the various organ involvements was calculated for both outcome groups.

\section{RESULTS}

From January 1985 through December 1995, 244 subjects met the eligibility criteria for post-intrapartum asphyxial HIE. Data enabling the evaluation of all four organs/systems additional to the central nervous system were available for 144 of these infants (59\%). Four infants lacked data on eligibility criterion 1 ; all four had intrapartal as well as neonatal courses indicative of severe intrapartum asphyxia. Contact could not be established in 14 cases.

Table 1 gives the basic characteristics for both outcome groups of infants. The five minute Apgar score was less than 5 in 89/141 infants with data. Spontaneous regular respiration was established after five minutes in 110/123 infants with data. Chest compression was performed for more than one minute in 51 infants. Seizures or coma were documented in $125(87 \%)$ infants (median age of onset four hours; interquartile range 2-8.5 hours; 119 infants had seizures before 24 hours of age). Another 15 infants were obtunded or lethargic (Sarnat stage 2), and four infants were irritable (Sarnat stage 1).

By the minimum age of 24 months, 80 of the 144 infants (55\%) had severe adverse outcomes, 50 (35\%) infants were free of severe adverse outcome, and $14(10 \%)$ infants had missing outcome data (of the remaining 100 infants not included for lack of organ/system assessment in this study, 47 (47\%) had adverse outcome, 41 (41\%) had good outcome, and $12(12 \%)$ had unknown outcome). Figure 1 shows the details of the outcomes.

All of the 130 infants with known outcomes showed evidence of at least one organ dysfunction in addition to brain. Renal, cardiovascular, pulmonary, and hepatic dysfunction was present in 91 (70\%), $80(62 \%), 112(86 \%)$, and $110(85 \%)$ infants respectively (table 2 ). No differences were observed in the rates of involvement of each organ according to outcome. Table 3 shows the relation between the number of additional organs involved and outcome. The rates of adverse outcomes increased as the number of additional organs involved increased from one to three, but decreased when an additional four organs were involved.

\begin{tabular}{llll} 
Table 1 & \multicolumn{3}{l}{ Basic characteristics of the outcome groups } \\
\hline \multicolumn{5}{c}{$\begin{array}{l}\text { Good outcome } \\
(\mathbf{n}=50)\end{array}$} & $\begin{array}{l}\text { outcome } \\
(\mathbf{n}=\mathbf{8 0})\end{array}$ & p Value \\
Variable & $40.2(1.6)$ & $39.9(1.6)$ & 0.34 \\
\hline Gestational age (weeks) & $3488(509)$ & $3420(500)$ & 0.41 \\
Birth weight (g) & $29.1(6.4)$ & $29.3(5.8)$ & 0.82 \\
Maternal age (years) & 2 & 2 & - \\
Gravida (median) & 0 & 0 & - \\
Para (median) & 4 & 3 & - \\
Five minute Apgar score & 4 & & \\
(median) & &
\end{tabular}




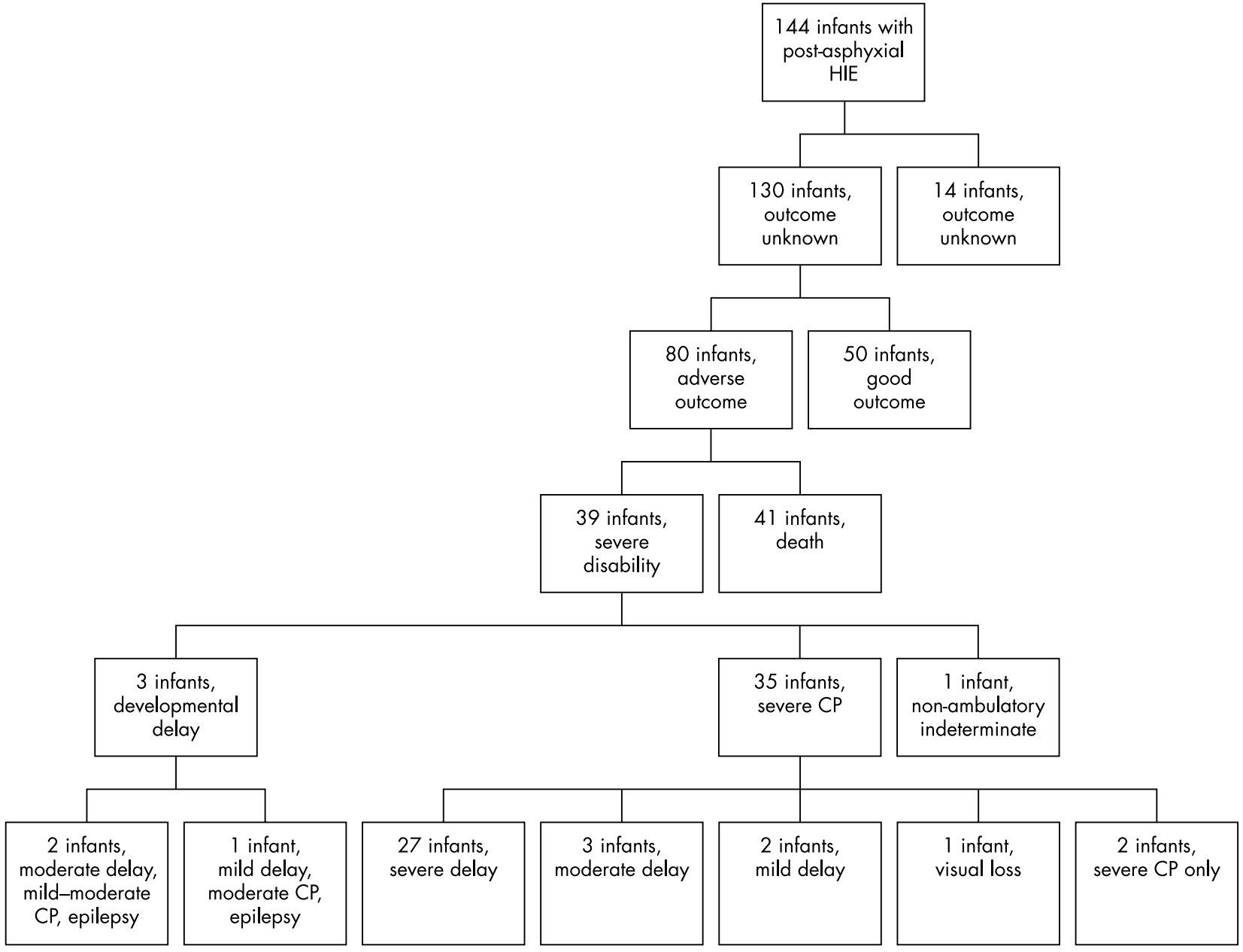

Figure 1 Outcome data of the study cohort. CP, Cerebral palsy.

\section{DISCUSSION}

All infants with severe post-asphyxial HIE had evidence of dysfunction of at least one organ/system in addition to the central nervous system. This conforms with the criteria of the American College of Obstetricians and Gynecologists ${ }^{2}$ and Society of Obstetricians and Gynaecologists of Canada, ${ }^{1}$ but not with some published reports of organ/system dysfunction in neonates with asphyxia of variable severity. ${ }^{71112}$ The variability in the reported incidence of MOD may be explained by $(a)$ the selection criteria for studies of MODat the mild end of the spectrum are cases of "intrapartum asphyxia" with or without HIE during the neonatal period, ${ }^{711} 12$ whereas at the severe end of the spectrum are known cases of cerebral palsy attributed to intrapartum asphyxia - and $(b)$ the differences in the definition of MOD with respect to the number of organs included in its definition, the definition of "organ/system" (for example,

Table 2 Organ involvement in relation to long term outcome

\begin{tabular}{llll}
\hline Organ & Good outcome & Adverse outcome & Total \\
\hline Renal & $30 / 50(60)$ & $61 / 80(76)$ & $91 / 130(70)$ \\
Cardiovascular & $29 / 50(58)$ & $51 / 80(64)$ & $80 / 130(62)$ \\
Pulmonary & $43 / 50(86)$ & $69 / 80(86)$ & $112 / 130(86)$ \\
Liver & $44 / 50(88)$ & $66 / 80(82)$ & $110 / 130(85)$ \\
\hline \multicolumn{4}{l}{ Values in parentheses are percentages. }
\end{tabular}

kidneys, hypocalcaemia), and the definition of dysfunction of each organ/system.

Although we confirmed evidence of MOD in all infants with severe intrapartum asphyxia, we did not find any relation between MOD and long term outcome. The recent consensus statement ${ }^{9}$ also suggested that MOD is a criterion to suggest intrapartum timing but is not a specific parameter. According to the concept of the diving reflex, dissonance would be expected between non-essential and essential organ/system dysfunction, particularly in the good outcome subgroup. Comparing the good and adverse outcomes groups, we found marginal differences in the incidences of kidney and cardiovascular system dysfunction but notably no differences in pulmonary and hepatic dysfunction. The rates of individual organ dysfunction varied from $64 \%$ to $86 \%$ for

$\begin{aligned} & \text { Table } 3 \\
& \text { involved and outcome }\end{aligned}$
\begin{tabular}{lll} 
Number of \\
$\begin{array}{l}\text { additional } \\
\text { organs } \\
\text { involved }\end{array}$ & $\begin{array}{l}\text { Good outcome } \\
(\mathbf{n}=50)\end{array}$ & $\begin{array}{l}\text { Adverse outcome } \\
(\mathbf{n}=80)\end{array}$ \\
\hline 1 & $5(10)$ & $1(1)$ \\
2 & $15(30)$ & $18(22)$ \\
3 & $9(18)$ & $34(43)$ \\
4 & $21(42)$ & $27(34)$ \\
\hline
\end{tabular}


infants with severe adverse outcome, and from 58\% to $86 \%$ for infants with good outcome. Thus, the proportion of infants with each individual organ/system involved does not distinguish infants in relation to long term outcome, suggesting that activation of the diving reflex was inconsistent.

Inconsistencies in organ involvement were also observed in animal experiments under controlled conditions. ${ }^{6}$ In a review Jensen $e^{2}$ al ${ }^{6}$ reported that, in experimental animals, different causes of asphyxia have different effects on blood flow to various organs. For example, maternal hypoxaemia, graded reduction of umbilical blood flow, and graded/repeated reduction of uterine blood flow were associated with activation of the diving reflex, whereas arrest of uterine blood flow reduced the blood flow to the brain. Our study included patients with all causes of asphyxia, which may explain the inconsistency in the activation of the diving reflex that we observed. Variation in individual vulnerability may also explain this variation. There was a stepwise increase in the rates of adverse outcomes with increase in the number of additional organs involved up to three organs, but the trend did not extend to four organs involved. This again suggests variable activation of the diving reflex.

The involvement of kidneys in $70 \%$ of cases in this cohort was comparable to the reported incidence in representative studies reflecting the spectrum of severity of asphyxia referred to above, although we chose the middle of the spectrum of published definitions..$^{82-14}$ The rate of cardiovascular system involvement in the present study (62\%) compares with the $50 \%$ rate reported by Shankaran et al ${ }^{12}$ and the $78 \%$ rate reported by Hankins et al. ${ }^{14}$ Despite different criteria for lung dysfunction, our result of $86 \%$ incidence of lung involvement concurs with the incidence reported by Shankaran $\mathrm{et} \mathrm{l}^{12}$ in a similar group of patients. Finally, using similar criteria to those of Phelan et $\mathrm{al}^{8}$ for liver involvement, we found an $84 \%$ rate compared with $23 \%$, whereas Hankins et al, ${ }^{14}$ using plus 2 SD to define abnormal values, reported a rate of $80 \%$. These differences in liver involvement may be explained by differences in timing of the measurements, as values return to normal within a few days of the asphyxial event in most infants. Many missing data and the use of whole case series as the denominator, as discussed by Hankins et al, ${ }^{14}$ may explain the discordance of the results of Phelan et al.

The criteria for determining the appropriate timing for evaluation of infants for MOD have not been fully studied. Data from the studies of normal and asphyxiated infants indicate that 24-72 hours after birth is most likely to capture biochemical and electrocardiographic abnormalities associated with asphyxia. ${ }^{16-21}$ Simultaneous monitoring of infants by cerebral function monitor (amplitude integrated electroencephalography) can provide useful information about the changes in the neurological status of these infants. ${ }^{22}$

The strengths of our study are the large sample size, the variability of outcomes, the high proportion of infants with known long term outcomes, the selection of study subjects for completeness of data on involvement of organs/systems, and the consistency of our results with those reported in the literature. As indicated above, Hankins et al ${ }^{14}$ reported similar rates of MOD in their 46 infants with neonatal encephalopathy; however, there were missing data in their study, and no correlations with long term outcomes were performed. Our study has the known limitations of retrospective studies. ${ }^{23}$

In conclusion, we found evidence in support of the MOD criterion in the definition of asphyxia, but not for identification of infants at risk of long term adverse outcome injury following severe intrapartum asphyxia. However, if MOD is to remain an essential criterion for intrapartum asphyxia, multiple organs/systems including the four in this study should be evaluated in each individual patient. In addition, the evaluation should be carried out at the most appropriate time for each criterion. For future prospective multicentre studies, there is clearly a need to develop a consensus of opinion about the definitions of organ dysfunction and the criteria for MOD.

\section{Authors' affiliations}

P Shah, J Beyene, Department of Paediatrics, Mount Sinai Hospital, and University of Toronto, Toronto, Ontario, Canada

S Riphagen, M Perlman, Division of Neonatology, Hospital for Sick Children, Toronto, and Department of Paediatrics, University of Toronto

\section{REFERENCES}

1 Policy statement: Task force on cerebral palsy and neonatal asphyxia (part 1). J SOGC 1996;18:1267-79.

2 ACOG Committee Opinion. Inappropriate uses of the terms fetal distress and birth asphyxia. Int J Gynaecol Obstet 1998;61:309-10.

3 Bocking AD, Gagnon R, White SE, et al. Circulatory responses to prolonged hypoxemia in fetal sheep. Am J Obstet Gynecol 1988;159:1418-24.

4 Peeters LL, Sheldon RE, Jones MD, et al. Blood flow to fetal organs as a function of arterial oxygen content. Am J Obstet Gynecol 1979;135:637-46.

5 Sheldon RE, Peeters LL, Jones MD, et al. Redistribution of cardiac output and oxygen delivery in the hypoxemic fetal lamb. Am J Obstet Gynecol 1979;135:1071-8.

6 Jensen A, Garnier Y, Berger R. Dynamics of fetal circulatory responses to hypoxia and asphyxia. Eur J Obstet Gynecol Reprod Biol 1999:84:155-72.

7 Martin-Ancel A, Garcia-Alix A, Gaya F, et al. Multiple organ involvement in perinatal asphyxia. J Pediatr 1995;127:786-93.

8 Phelan JP, Ahn MO, Korst L, et al. Intrapartum fetal asphyxial brain injury with absent multiorgan system dysfunction. J Matern Fetal Med 1998;7:19-22.

9 MacLennan A. A template for defining a causal relation between acute intrapartum events and cerebral palsy: international consensus statement. BMJ 1999;319:1054-9.

10 Volpe JJ. Neonatal seizures: current concepts and revised classification. Pediatrics 1989;84:422-8.

11 Perlman JM, Tack ED, Martin T, et al. Acute systemic organ injury in term infants after asphyxia. American Journal of Diseases in Children 1989;143:617-20.

12 Shankaran S, Woldt E, Koepke T, et al. Acute neonatal morbidity and longterm central nervous system sequelae of perinatal asphyxia in term infants. Early Hum Dev 1991;25:135-48.

13 Perlman JM, Tack E, eds. Renal injury in the asphyxiated newborn infant: relationship to neurologic outcome. J Pediatr 1988;113:875-9.

14 Hankins GD, Koen S, Gei AF, et al. Neonatal organ system injury in acute birth asphyxia sufficient to result in neonatal encephalopathy. Obstet Gynecol 2002;99:688-91.

15 Chauhan SP, Magann EF, Morrison JC. Neonatal organ system injury in acute birth asphyxia sufficient to result in neonatal encephalopathy. Obstet Gynecol 2003;101:203-4.

16 Bueva A, Guignard JP. Renal function in preterm neonates. Pediatr Res 1994;36:572-7.

17 Gordjani N, Burghard R, Leititis JU, et al. Serum creatinine and creatinine clearance in healthy neonates and prematures during the first 10 days of life. Eur J Pediatr 1988;148:143-5.

18 Jedeikin R, Primhak A, Shennan AT, et al. Serial electrocardiographic changes in healthy and stressed neonates. Arch Dis Child 1983;58:605-11.

19 Primhak RA, Jedeikin R, Ellis G, et al. Myocardial ischaemia in asphyxia neonatorum. Electrocardiographic, enzymatic and histological correlations. Acta Paediatr Scand 1985;74:595-600.

20 Lackmann GM, Tollner U. The predictive value of elevation in specific serum enzymes for subsequent development of hypoxic-ischemic encephalopathy or intraventricular hemorrhage in full-term and premature asphyxiated newborns. Neuropediatrics 1995;26:192-8.

21 Zanardo V, Bondio M, Perini G, et al. Serum glutamic-oxaloacetic transaminase and glutamic-pyruvic transaminase activity in premature and full-term asphyxiated newborns. Biol Neonate 1985:47:61-9.

22 Hellstrom-Westas L, Rosen I, Svenningsen NW. Predictive value of early continuous amplitude integrated EEG recordings on outcome after severe birth asphyxia in full term infants. Arch Dis Child Fetal Neonatal Ed 1995; 72:F34-8.

23 Sacks H, Chalmers TC, Smith H Jr. Randomized versus historical controls for clinical trials. Am J Med 1982;72:233-40. 